ISSN 0103-5150

Fisioter. Mov., Curitiba, v. 25, n. 1, p. 127-140, jan./mar. 2012 Licenciado sob uma Licença Creative Commons

\title{
Eletrolipólise por meio da estimulação nervosa elétrica transcutânea (Tens) na região abdominal em pacientes sedentárias e ativas
}

\author{
Eletrolipólise through electric transcutaneous nerve stimulation \\ (Tens) in the abdominal region in sedentary patients and active
}

\author{
Natália Ribeiro de Melo ${ }^{[a]}$, Flávia Maria de Albuquerque Cordeiro Monteiro ${ }^{[a]}$, \\ Gabriela Arruda Reinaux Pontes ${ }^{[b]}$ Simone Monte Bandeira de Mello ${ }^{[c]}$ \\ [a] Fisioterapeuta pela Faculdade do Agreste de Pernambuco (ASCES), Caruaru, PE - Brasil \\ [b] Fisioterapeuta, Especialista em Fisioterapia Pediátrica e Neonatal, Caruaru, PE - Brasil, e-mail: garv.fisio@gmail.com \\ [c] Professora da ASCES, Universo, fisioterapeuta, Especialista em Dermato-Funcional, Recife, PE - Brasil
}

Resumo

Introdução: A eletrolipólise é uma técnica destinada ao tratamento de acúmulo de tecido adiposo localizado em determinadas regiões. Sua aplicação ocorre por meio de pares de agulhas de acupuntura no tecido subcutâneo, ligadas a uma corrente de baixa frequência. Essa estimulação elétrica provoca diversas modificações fisiológicas no adipócito, dentre elas o incremento do fluxo sanguíneo local, aumento do metabolismo celular e queima de calorias. Objetivos: Este estudo teve como objetivo avaliar o efeito da eletrolipólise na redução de gordura localizada na região infra-abdominal, em pacientes que praticam atividade física e nas sedentárias. Materiais e métodos: As pacientes na faixa etária dos 18 aos 30 anos, sexo feminino, foram submetidas à avaliação em que foi medido o índice de massa corpórea (IMC) e a razão cintura/quadril (C/Q). Com uma fita métrica foi realizada a perimetria de cintura (abaixo da última costela), da crista ilíaca, $5 \mathrm{~cm}$ e $10 \mathrm{~cm}$ acima da crista ilíaca. Foram realizadas 20 sessões, sendo duas vezes por semana, com oito agulhas de acupuntura conectadas a um aparelho de estimulação transcutânea de corrente de baixa frequência (Tens). Resultados: Foi observada a redução de medidas do tecido adiposo em ambos os grupos, havendo maior notoriedade no grupo das sedentárias. Conclusão: Apesar da significativa perda de tecido adiposo neste estudo, os resultados não foram expressivos pela desistência 
das voluntárias no decorrer do tratamento, sendo esse um ponto bastante dificultoso do trabalho, que deveria ter permanecido com as 26 pacientes.

Palavras-chave: Eletrolipólise. Tens. Metabolismo.

\section{Abstract}

Introduction: Electrolipolysis is a thechnique used to treat the accumulation of fat tissue located in certain regions of the body. It's application occurs through a pair of acunpucture needles into the subcutaneous tissue connected to a low-frequency current. This electrical stimulation causes several physiological changes in the adipocyte, among them, the increase in local blood flow, increased cellular metabolism and burn calories. objectives: To evaluate the effect of electrolipolysis in reducing localized fat in the infra-abdominal in patients who engage in physical activity and in sedentary patients. Materials and methods: Patients aged within 18 an 30 years, female, underwent evaluation where we measured the body mass index (BMI), waist/hip ratio (W/H). With a tape measure it was measured the waist circumference (below the last rib), the lower abdomen (above the iliac crest), $5 \mathrm{~cm}$ and $10 \mathrm{~cm}$ above the lower abdomen. The patients underwent 20 sessions, twice a week with eight acupuncture needles connected to a transcutaneous electric stimulation of low-frequency current (Tens). Results: It was observed the reduction of measures of fat tissue in both groups, being most noticeable in the sedentary group. Conclusion: Despite the significant loss of fat in this study, the results were not significant because of the withdrawal of volunteers during the treatment. This was actually a point that made the study more difficult, as the work should have been done with the 26 patients.

Keywords: Electrolipolysis. Tens. Metabolism.

\section{Introdução}

Quando há excesso de ingestão calórica, este é estocado na forma de gordura, geralmente responsável pela formação da gordura localizada, como depósitos resistentes ao emagrecimento, codificados geneticamente, e que mantêm as características familiares (1-3). Existem dois tipos de depósitos de gordura: os depósitos gerais (mobilizam-se facilmente) e os depósitos hereditários (resistentes ao emagrecimento) (2).

0 padrão de distribuição de gordura é hereditário e pode ser associado à atividade regional da enzima denominada lipase lipoproteica, que limita o ritmo de captação dos triglicerídeos pela célula adiposa (1). Duas enzimas lipolíticas principais, LSH (lipase-sensível a hormônio) e LPL (lipoproteína lipase), atuam respectivamente no interior do adipócito e nas lipoproteínas ricas em triglicérides (TG) (3-4).

A estimulação elétrica subcutânea abdominal é um procedimento frequentemente utilizado para a aceleração do metabolismo gorduroso. A transcutâneous eletrical nerve stimulation (Tens) é um estimulador elétrico com a capacidade de estimular nervos periféricos com eletrodos colocados na superfície do corpo, onde geram pulsos de tensão (5).

A eletrolipólise é uma técnica que utiliza a estimulação elétrica, destinada ao tratamento das adiposidades localizadas. Essa aplicação ocorre por meio de vários pares de agulhas de acupuntura no tecido subcutâneo, ligados à corrente de baixa intensidade. Tal estimulação elétrica provoca diversas modificações fisiológicas no adipócito, dentre elas, o incremento do fluxo sanguíneo local, aumentando o metabolismo celular e facilitando a queima de calorias (6-7).

Com a utilização da Tens, no tratamento de desordens similares no organismo, bons resultados são obtidos. Sendo assim, é natural que seja mais um recurso fisioterapêutico utilizado para o tratamento das adiposidades abdominais, pois a estimulação elétrica subcutânea abdominal é um procedimento frequentemente utilizado para a aceleração do metabolismo gorduroso (8-11).

Com a carência de estudo científico nessa área surgiu o interesse de realizar uma pesquisa para observação da aplicação da Tens como um recurso de eletrolipólise em pacientes com gordura localizada 
em abdômen. No entanto, este estudo pretende comparar o efeito da estimulação elétrica no metabolismo adiposo ao se comparar dois grupos de mulheres distintas: um grupo com pacientes praticantes de atividades físicas; e o outro com pacientes sedentárias. Dessa forma, analisar-se-á a influência do exercício físico associado à estimulação elétrica (12-16).

\section{Materiais e métodos}

O estudo foi realizado na Clínica Escola de Fisioterapia, localizada na Faculdade do Agreste de Pernambuco (Faape) e Associação Caruaruense de Ensino Superior de Caruaru (ASCES).

Metodologicamente, caracterizou-se como sendo um estudo de intervenção, não randomizado e sem grupo controle, em que foi comparado o efeito de uma intervenção (corrente de eletroestimulação Tens), analisando os resultados encontrados entre os dois grupos que apresentaram características distintas (prática ou não de atividade física).

0 estudo teve como amostra 26 voluntárias do sexo feminino, com idade entre 18 e 30 anos, randomicamente selecionadas por conveniência. Tais voluntárias foram divididas em dois grupos com 12 participantes cada, em que o Grupo A foi o das mulheres que realizam alguma atividade física três vezes por semana, sendo aplicado o Questionário de Baecke, e o Grupo B foi o das mulheres classificadas como sedentárias.

Foram incluídas mulheres com queixa de adiposidade localizada na região infra-abdominal. Foram excluídas as pacientes em uso de medicamentos, portadoras de doenças cardíacas e diabetes, obesas, com lesões na pele, com tumoração de natureza maligna, em tratamento com corticoides e progesterona prolongados, com mioma uterino e implantes metálicos, órteses, próteses ou fios.

As voluntárias foram submetidas à avaliação por meio da coleta de dados, em que foi medido o índice de massa corpórea (IMC), determinado pela razão do peso pela altura ao quadrado $\left(\mathrm{kg} / \mathrm{m}_{2}\right)$, e a razão cintura/quadril (C/Q) pelo quociente entre as perimetrias da cintura e quadril. Com uma fita métrica não elástica foi realizada a perimetria de cintura (abaixo da última costela), crista ilíaca $5 \mathrm{~cm}$ e 10 $\mathrm{cm}$ acima da crista ilíaca. Assim como, também, foi avaliado o peso corporal com a Balança Slim taxa de gordura MEA-02510. As praticantes de atividades físicas foram submetidas ao questionário voltado à estimativa do nível de prática habitual de atividade física proposto por Baecke, estruturado de forma quali-quantitativa em escala de Likert $(7,20)$.

As participantes da pesquisa foram fotografadas antes de começarem o tratamento por uma máquina fotográfica Sony DSC-W210 em uma distância de $40 \mathrm{~cm}$, após dez sessões e ao fim da pesquisa.

0 tratamento foi composto de 20 sessões, sendo realizadas duas sessões por semana, na Clínica Escola da Faculdade do Agreste de Pernambuco. 0 aparelho da Tens Physiotonus II TENS + FES Bioset foi utilizado com a corrente Tens, tendo a intensidade de acordo com a sensibilidade da paciente, em que o limite máximo chegou apenas a $70 \mathrm{~mA}$. Cada canal foi subdividido em dois canais, dessa forma passando a ter quatro canais. As agulhas de acupuntura de $0,25 \times 30$ e descartáveis foram dispostas $6 \mathrm{~cm}$ abaixo da cicatriz umbilical, colocadas paralelamente, distando entre elas $5 \mathrm{~cm}$ com $1 \mathrm{~cm}$ de diferença.

Os dados foram coletados por meio da avaliação física de cada paciente antes do tratamento, após dez sessões e ao fim do tratamento, em que foram processados por meio do statistical package for the social sciences (SPSS) (19).

Assim, os resultados encontrados foram submetidos à análise de variância (19), uma análise que compara as médias obtidas nas diferentes amostras.

\section{Resultados}

0 estudo foi iniciado com 26 pacientes, das quais 13 eram sedentárias e 13 praticantes de atividade física. Entretanto, no decorrer do estudo houve desistências: 14 voluntárias não concluíram as dez primeiras sessões, dessa forma apenas 12 foram reavaliadas após a décima sessão. As 20 sessões foram concluídas apenas por 8 voluntárias.

Foi constatado que em todos os casos as não sedentárias possuíam medidas maiores que as das sedentárias, e permaneceram dessa forma. Nos quesitos peso e crista ilíaca, as não sedentárias tiveram, em média, aumento nas medidas. Em todos os outros casos, houve decréscimo nas medidas, tanto para as sedentárias quanto para as não sedentárias. Em termos percentuais, a melhoria sempre foi mais acentuada no caso das pacientes sedentárias, sendo a maior perda percentual de 6,75\% na cintura das sedentárias. 
Na sequência, seguem os gráficos que ilustram os valores médios das medidas (peso e perimetria) das pacientes. 0 Gráfico 1 representa, na ordenada, os valores dos pesos das pacientes em quilograma $(\mathrm{kg})$ e, nas abscissas, a quantidades de sessões realizadas. Observou-se que as pacientes sedentárias obtiveram diminuição do peso até dez sessões e o mantiveram até o fim do tratamento. Entretanto, as pacientes não sedentárias mantiveram o peso inicial durante todo o tratamento.

0 Gráfico 2 representa, na ordenada, os valores da perimetria das cinturas das pacientes em centímetros (cm) e, nas abscissas, a quantidade de sessões realizadas. Observou-se que as pacientes sedentárias obtiveram maior redução de medidas até dez sessões e se mantiveram assim até o fim do tratamento. Entretanto, nas pacientes não sedentárias a perda significante foi após as 20 sessões.

0 Gráfico 3 representa, na ordenada, os valores da perimetria das cristas ilíacas das pacientes em centímetros $(\mathrm{cm})$ e, nas abscissas, a quantidade de sessões realizadas. A perimetria teve como ponto de referência a crista ilíaca, e as medidas foram realizadas acima da marca na avaliação inicial, após 10 sessões e ao fim das 20 sessões. Observou-se que as pacientes sedentárias obtiveram maior redução de medidas até dez sessões e a mantiveram até o fim do tratamento. Entretanto, nas pacientes não sedentárias percebeu-se aumento dessas medidas.

0 Gráfico 4 representa, na ordenada, os valores da perimetria 5 centímetros acima das cristas ilíacas das pacientes e, nas abscissas, a quantidade de sessões

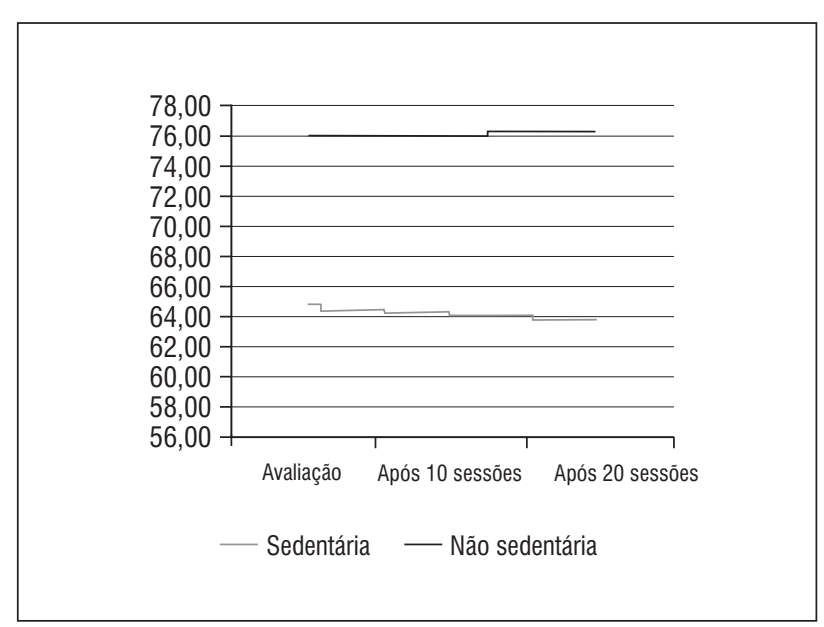

Gráfico 1 - Comparação dos valores dos pesos em quilograma (kg) das mulheres sedentárias e não sedentárias Fonte: Dados da pesquisa.

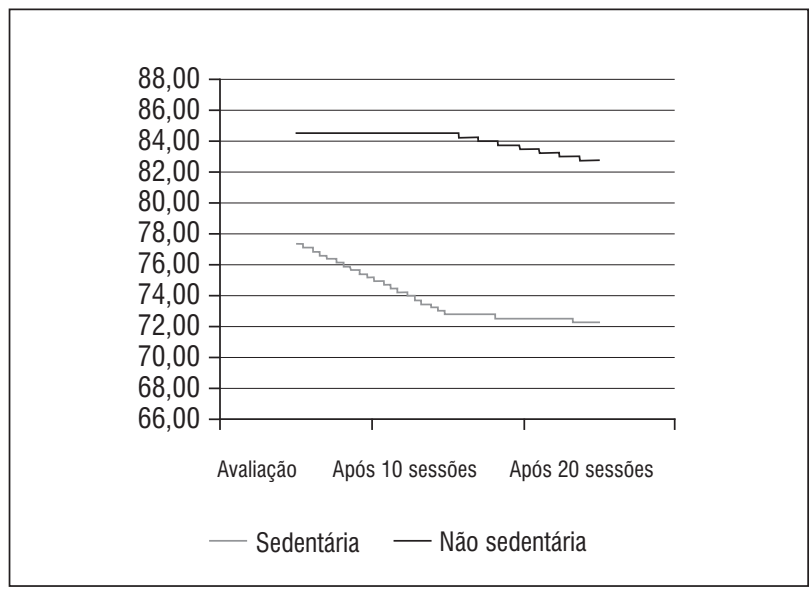

Gráfico 2 - Comparação dos valores da perimetria das cinturas em centímetros (cm) das mulheres sedentárias e não sedentárias

Fonte: Dados da pesquisa.

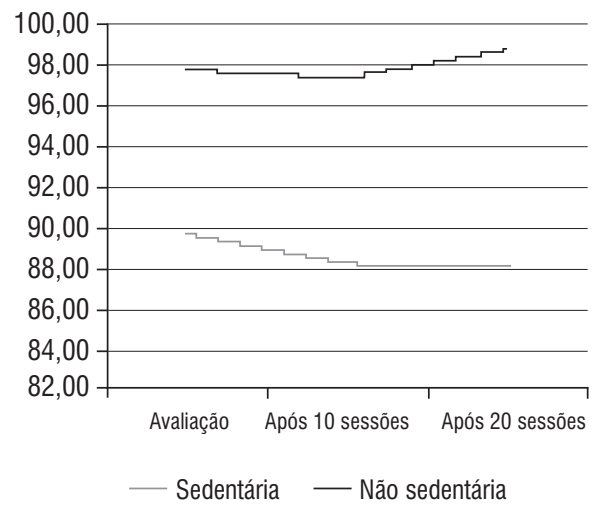

Gráfico 3 - Comparação dos valores da perimetria da crista ilíaca das mulheres sedentárias e não sedentárias

Fonte: Dados da pesquisa.

realizadas. Foi realizada a perimetria de $5 \mathrm{~cm}$ acima da crista ilíaca na avaliação inicial, após dez sessões e na avaliação final. Observou-se que ambos os grupos obtiveram decréscimo das medidas.

0 Gráfico 5 representa, na ordenada, os valores da perimetria $10 \mathrm{~cm}$ acima das cristas ilíacas das pacientes e, na abscissa, a quantidade de sessões realizadas. Foi realizada a perimetria de $10 \mathrm{~cm}$ acima da crista ilíaca na avaliação inicial, após dez sessões e na avaliação final. Observou-se que ambos os grupos obtiveram decréscimo das medidas.

A Tabela 1, apresentada na sequência, representa os valores demonstrados nos gráficos anteriores, 


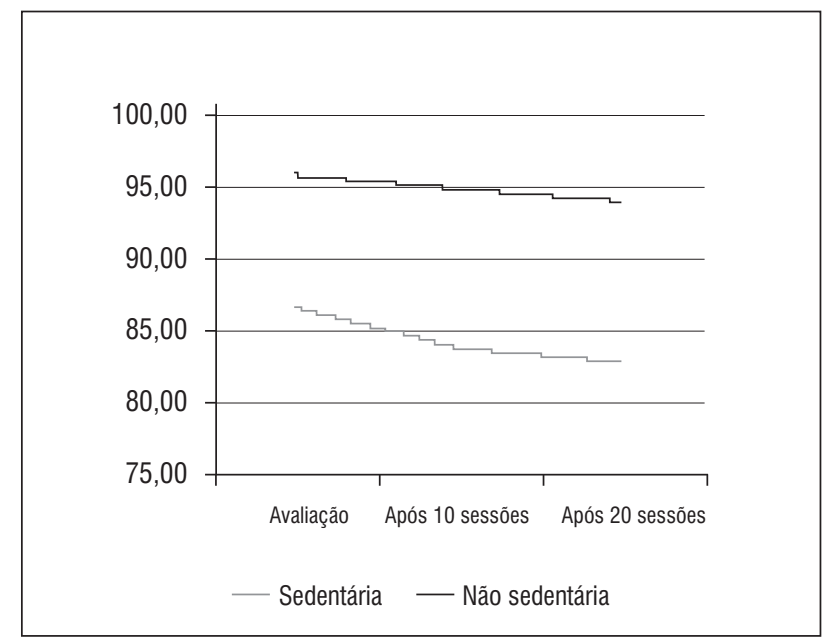

Gráfico 4 - Comparação dos valores da perimetria $5 \mathrm{~cm}$ acima das cristas ilíacas das mulheres sedentárias e não sedentárias

Fonte: Dados da pesquisa.

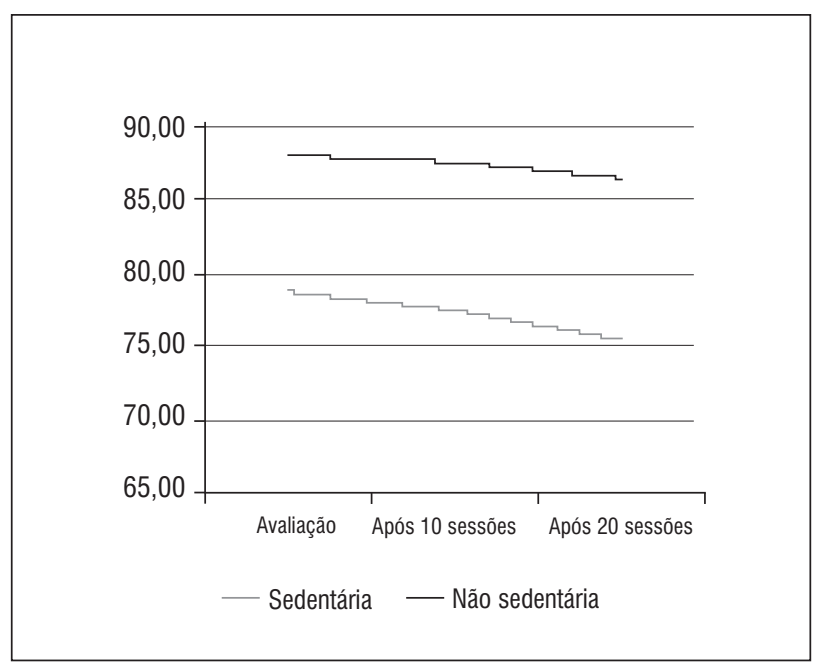

Gráfico 5 - Comparação dos valores da perimetria $10 \mathrm{~cm}$ acima das cristas ilíacas das mulheres sedentárias e não sedentárias

Fonte: Dados da pesquisa.

ressaltando (na última coluna) a diferença percentual entre o valor médio da medida no início do tratamento e o valor médio da medida após as 20 seções do tratamento.

Esses resultados foram submetidos a uma análise de variância (19) (ANOVA não paramétrico), realizada a partir da ferramenta estatística SPSS (19), sobre as perdas das medidas das pacientes, revelando a tendência de um possível aumento na medida do quadril das pacientes não sedentárias.

\section{Discussão}

Durante o exercício, a demanda energética do músculo esquelético aumenta, consumindo uma quantidade maior de trifosfato de adenosina (ATP). Existem três processos distintos e integrados que operam para satisfazer a demanda energética do músculo: o sistema anaeróbio, que pode ser dividido em aláctico e láctico; o sistema aláctico, que compreende a quebra da creatina fosfato (CP) e as moléculas de ATP já presentes dentro do músculo; já o sistema lático refere-se à combustão parcial da glicose ou glicogênio. A quebra dessas duas moléculas irá gerar ácido láctico com a sua imediata conversão para lactato. E, finalmente, o sistema aeróbio, que se refere à combustão completa dos carboidratos (glicose e glicogênio), gorduras e em alguns casos proteínas na presença do oxigênio (17-20).

A quantidade de energia que é obtida pelo mecanismo das gorduras e das proteínas representa cerca de $80 \%$ da energia total obtida pelas células, em comparação com os $20 \%$ obtidos da oxidação da glicose. Em razão disso, metade ou mais dos carboidratos ingeridos por uma pessoa são primeiro armazenados em forma de gordura e depois utilizados para a energia, em forma de ácidos graxos (21- 29).

A ação da LSH causa a liberação do glicerol (glicerol livre) e dos ácidos graxos livres (AGL) na circulação sistêmica. A LPL atua liberando glicerol e ácido graxo (AG) na circulação, principalmente pela ação sobre as VLDL (lipoproteínas de densidade muito baixas). 0 AG é captado pelo tecido adiposo produzindo triglicérides (TGAG). Os AGL são captados pelo músculo e pelo fígado para serem oxidados (obtenção energia), parcialmente oxidados (produção de corpos cetônicos) ou reesterificados para formarem triglicérides (TG-AG) novamente (3). A Tens proporciona semiondas quadradas positivas com pico negativo que estimulam os receptores nervosos ao mesmo tempo em que impedem a acomodação. Esses impulsos também incrementam o fluxo de sangue e eliminam os elementos de dejetos $(9,18,24,26)$. Assumpção (21) relata que a eletrolipólise deve ser realizada por meio de aparelhos que emitem correntes de baixa frequência (21).

Alguns autores relatam que a eletrolipólise é uma técnica destinada ao tratamento das adiposidades localizadas, por meio da aplicação de vários pares de agulhas de acupuntura no tecido subcutâneo, ligadas à corrente de baixa intensidade. A estimulação 
Tabela 1 - Diferenças entre as medidas antes e após as sessões de eletrolipólise nas mulheres sedentárias e não sedentárias

\begin{tabular}{|c|c|c|c|c|}
\hline Grupo & Peso inicial & Peso após 10 sessões & Peso após 20 sessões & Diferença (\%) \\
\hline Sedentárias & 63,58 & 63,05 & 64,13 & 0,85 \\
\hline Não sedentárias & 71,87 & 72,00 & 76,40 & 6,31 \\
\hline Grupo & Cintura inicial & Cintura após 10 sessões & Cintura após 20 sessões & Diferença(\%) \\
\hline Sedentárias & 77,75 & 73,00 & 72,50 & $-6,75$ \\
\hline Não sedentárias & 85,13 & 85,00 & 83,13 & $-2,35$ \\
\hline Grupo & Crista ilíaca inicial & Crista ilíaca 10 sessões & Crista ilíaca 20 sessões & Diferença (\%) \\
\hline Sedentárias & 89,75 & 88,25 & 88,00 & $-1,95$ \\
\hline Não sedentárias & 97,88 & 97,50 & 98,88 & 1,02 \\
\hline Grupo & $\begin{array}{l}5 \mathrm{~cm} \text { acima da Crista } \\
\text { ilíaca inicial }\end{array}$ & $\begin{array}{l}5 \mathrm{~cm} \text { acima da Crista } \\
\text { ilíaca após } 10 \text { sessões }\end{array}$ & $\begin{array}{l}5 \mathrm{~cm} \text { acima da Crista } \\
\text { ilíaca após } 20 \text { sessões }\end{array}$ & Diferença (\%) \\
\hline Sedentárias & 86,38 & 83,63 & 82,63 & $-4,34$ \\
\hline Não sedentárias & 95,50 & 94,50 & 93,75 & $-1,83$ \\
\hline Grupo & $\begin{array}{l}10 \mathrm{~cm} \text { acima da Crista } \\
\text { ilíaca inicial }\end{array}$ & $\begin{array}{l}10 \mathrm{~cm} \text { acima da Crista } \\
\text { ilíaca após } 10 \text { sessões }\end{array}$ & $\begin{array}{l}10 \mathrm{~cm} \text { acima da Crista } \\
\text { ilíaca após } 20 \text { sessões }\end{array}$ & Diferença (\%) \\
\hline Sedentárias & 78,75 & 77,50 & 75,38 & $-4,28$ \\
\hline Não sedentárias & 88,13 & 87,63 & 86,63 & $-1,70$ \\
\hline
\end{tabular}

Fonte: Dados da pesquisa.

elétrica provoca diversas modificações fisiológicas no adipócito, dentre elas o incremento do fluxo sanguíneo local, aumentando o metabolismo celular e facilitando a queima de calorias (30-33).

0 efeito Joule, presente na corrente elétrica, ao circular por um condutor proporciona certo tipo de calor. Esse aumento de temperatura não atinge tecidos orgânicos, visto que se trata de uma corrente com intensidade muito pequena. No entanto, é o suficiente para promover uma vasodilatação com aumento de fluxo sanguíneo local, com esse efeito já estimulado do metabolismo celular local, facilitando a queima de calorias e melhorando o trofismo celular $(10,18,21)$.

0 efeito neuro-hormonal, quando se utiliza uma corrente específica de baixa frequência durante a eletrolipólise, produz estimulação artificial do sistema nervoso simpático e, como consequência, ocorre a liberação de catecolaminas com aumento do AMP cíclico intradipocitário, e aumento da hidrólise dos triglicerídeos $(14,17,18)$.

Autores afirmam que a frequência pode variar entre 5 e $500 \mathrm{~Hz}$; no entanto, em outro estudos foi observado que esses valores devem ser mais baixos, em torno de 5 a $50 \mathrm{~Hz}$. Em relação à intensidade, alguns estudos sugerem que esta deve ser abaixo de $1 \mathrm{~mA}$; no entanto, foi visto que há relatos de que se deve utilizar de $2 \mathrm{~mA}$ a $10 \mathrm{~mA}$, e outros sugerem de $5 \mathrm{~mA}$ a $40 \mathrm{~mA}$ (10-12).

Como o Tens é uma corrente com efeito de acomodação, a intensidade deve ser ajustada de acordo com a sensibilidade de cada paciente (10-12). Neste estudo a intensidade utilizada estava na faixa de 1 $\mathrm{mA}$ até $70 \mathrm{~mA}$. Entretanto, observou-se que as pacientes que atingiam intensidades mais elevadas obtiveram redução mais significativa.

A eletrolipólise, também chamada de eletrolipoforese, é uma técnica destinada ao tratamento das adiposidades e acúmulo de ácidos graxos localizados. Caracteriza-se pela aplicação de microcorrente específica de baixa frequência (ao redor de $25 \mathrm{~Hz}$ ), que atua diretamente no nível dos adipócitos e dos lipídios acumulados, produzindo sua destruição e favorecendo sua posterior eliminação $(10,14,18)$.

Observou-se que a estimulação elétrica do tecido adiposo com eletrodos subcutâneos reduziu 
significativamente as medidas de abdômen e cintura, não alterou as concentrações séricas do perfil lipídico e promoveu redução nos níveis de glicerol livre após seis aplicações do procedimento. 0 estudo em questão concorda com esta pesquisa, em que foi observada a redução de medidas do tecido adiposo em ambos os grupos, havendo maior notoriedade no grupo das sedentárias. Possivelmente, em razão do maior percentual de gordura desse grupo (3).

Os resultados deste estudo comprovam a eficácia da eletrolipólise na redução de tecido adiposo da região abdominal. Mesmo com o número reduzido de pacientes que concluíram o tratamento (quatro pacientes sedentárias e quatro pacientes não sedentárias), foi possível observar a redução de $6,75 \%$ das medidas, porém houve aumento das medidas de crista ilíaca na pacientes praticantes de atividade física.

Apesar de ter obtido bons resultados da perda do tecido adiposo, esses resultados não foram expressivos pela desistência das voluntárias durante o tratamento. Tal situação comprometeu bastante a credibilidade desta pesquisa, a qual deveria ter sido concluída com as 26 pacientes.

Sugere-se que um novo estudo seja realizado, observando-se o real teor de gordura por meio de mensurações mais exatas, qual o tipo de atividade física praticada e a sua relação com o aumento de medidas (massa muscular), para que se obtenha resultados mais precisos.

\section{Referências}

1. Guirro ECO, Guirro RRJ. Fisioterapia dermato-funcional: fundamentos, recursos, patologias. São Paulo: Manole; 2005.

2. Neves SR, Oliveira D. Eficácia da associação de técnicas manuais e eletrotermoterapia na redução de medidas do abdome. Rev Biol e Saúde da UNISEP. 2007;1(1):67-71.

3. Paula RM, Simões NP, Picheth G. Efeitos da eletrolipoforese nas concentrações séricas do glicerol do perfil lipídico. Fisioter Bras. 2007;(3)5-9.

4. Kakeshita IS, Almeida SS. Relação entre índice de massa corporal e a percepção da auto-imagem em universitários. Rev Saúde Pública. 2006;40(3):497-504.
5. Tonella RM, Araújo S, Silva ÁMO. Estimulação elétrica nervosa transcutânea (TENS) no alivio da dor pós-operatório relacionada a procedimentos fisioterapêuticos em pacientes submetidos a cirurgias abdominais. Rev Brasil Anestesiol. 2006;56(6):630-42.

6. Garcia PG, Garcia FG, Borges FDS. O uso da eletrolipólise na correção de assimetria no contorno corporal pós-lipoaspiração: relato de caso. Rev Fisioter. Ser. 2005;5(8):20-1.

7. Florindo AAM, Latorre MRDO, Jaime PC, Tanaka T, Zerbini CAF. Metodologia para a avaliação da atividade física habitual em homens com 50 anos ou mais. Rev Saúde Pública. 2004;38(2):307-14.

8. Carneiro CH, Araújo DR, Santos FP, Neto JMV, Marino MAP, Mendonça NO, et al. Corpo, estética e obesidade: reflexões baseadas no paradigma da indústria cultural. Estudos. 2006;33(10):789-812.

9. Agnes JE. Eletrotermoterapia: teoria e prática. São Paulo: Oriun; 2005.

10. Soriano MCD, Pérez SC, Baques MIC. Electroestética profissional aplicada: teoria y práctica para la utilización de corrientes em estética. Espanha: Sorisa; 2000.

11. Parienti IJ. Medicina estética. São Paulo: Andrei; 2001.

12. Zaragoza JR, Rodrigo P. Eletroestética. Espanha: Nueva Estética; 1995.

13. Berg JM, Tymoczko JL, Stryer L. Bioquímica. São Paulo: Guanabara Koogan; 2005

14. Borges FS. Dermato-funcional: modalidades terapêuticas nas disfunções estéticas. São Paulo: Phorte; 2006.

15. Kitchen S. Eletroterapia: prática baseada em evidências. São Paulo: Manole; 2005.

16. Neves SR, Oliveira D. Eficácia da associação de técnicas manuais e eletrotermoterapia na redução de medidas do abdome. Rev Biol Saude Unisep. 2008;1(1):67-71.

17. Rodrigues BA, Santarém JM, Jacob W Filho, Meirelles ES, Nunes Marucci MF. Comparação da gordura corporal de mulheres idosas segundo antropometria, bioimpedância e DEXA. Arch Latinoam Nutr. 2001;51(1):49-56. 
18. Scorza FA, Figueiredo MM, Liao CO, Borges FS. Estudo comparativo dos efeitos da eletrolipólise com uso de TENS modo Burst e modo normal no tratamento de adiposidade localizada abdominal. Rev Ensaios e Ciência: Ciênc Biológ Agrárias e da Saúde. 2008;12(2):49-62.

19. Cabrera MAS. Relação do índice de massa corporal, da relação cintura-quadril e da circunferência abdominal com a mortalidade em mulheres idosas: seguimento de 5 anos. Cad Saúde Pública. 2005;21(3):767-75.

20. Guedes DP, Guedes JERP. Manual prático para avaliação em educação física. São Paulo: Manole; 2006.

21. Assumpção AC, Souza A, Máximo A, Cardoso MC, Borges FS. Eletrolipólise (eletrolipoforese) In: Borges FS. Fisioterapia dermato-funcional: modalidades terapêuticas nas disfunções estéticas. São Paulo: Phorte; 2006. p. 216-217.

22. Fonseca-Alaniz MH, Takada J, Alonso-Vale MIC, Lima FB. O tecido adiposo como centro regulador do metabolismo. Arq Bras Endocrinol Metab. 2006;50(2):216-29.

23. Glaner MF. Índice de massa corporal como indicativo da gordura corporal comparado às dobras cutâneas. Rev Bras Med Esporte. 2005;11(4):243-6.

24. Starkey C. Recursos terapêuticos em fisioterapia. São Paulo: Manole; 2001.

25. Navarro AM, Stedille MS, Unamuno MRL, Marchini JS. Distribuição da gordura corporal em pacientes com e sem doenças crônicas: uso da relação cintura-quadril e do índice de gordura do braço. Rev Nutr. 2001;14(1):37-41.
26. Kitchen S. Eletroterapia: prática baseada em evidências. São Paulo: Manole; 2003.

27. Santos M, Novaes P, França E, Cunha RM, Maia M. Os efeitos do treinamento intervalado e do treinamento contínuo na redução da composição corporal em mulheres adultas. [acesso 12 jul. 2011]. Disponível em: http://www.saudeemmovimento.com.br/revista/artigos/vida_e_saude/v2n2a6.pdf.

28. Chaves CRMM, Oliveira CQ Britto JAA, Elsas MICG. Exercício aeróbico, treinamento de força muscular e testes de aptidão física para adolescentes com fibrose cística: revisão da literatura. Rev Bras Saúde Matern Infant. 2007;7(3):245-50.

29. Fernandez AC, Mello MT, Tufik S, Castro PM, Fisberg M. Influência do treinamento aeróbio e anaeróbio na massa de gordura corporal de adolescentes obesos. Rev Bras Med Esporte. 2004;10(3):152-8.

30. Lerario DDG, Gimeno SG, Franco LJ, Iunes $M$, Ferreira SRG, Grupo de estudo de diabetes na comunidade nipo-brasileira, São Paulo, SP, Brasil. Excesso de peso e gordura abdominal para a síndrome metabólica em nipo-brasileiros. Rev Saúde Pública. 2002;36(1):4-11.
Recebido: $24 / 05 / 2011$

Received: 05/24/2011

Aprovado: 19/07/2011

Approved: 07/19/2011 


\section{Anexo 1}

\section{Termo de Consentimento Livre e Esclarecido para Participação}

Título: Utilização da estimulação nervosa elétrica transcutânea (Tens) na eletrolipólise na região do abdome em pacientes que realizam atividades físicas e pacientes sedentárias

\section{Investigadores:}

Nomes: Natália Ribeiro de Melo e Flávia Maria de Albuquerque Cordeiro Monteiro

Local de estudo: Clínica Escola da Faculdade do Agreste de Pernambuco

Este termo de consentimento pode conter palavras que você não entenda. Qualquer dúvida será esclarecida quando houver quaisquer palavras ou informações que você não entenda claramente.

Este estudo será realizado na Clínica Escola da Faculdade do Agreste de Pernambuco e pretende comparar o efeito da estimulação elétrica no metabolismo adiposo em dois grupos de mulheres distintas, um grupo com pacientes praticantes de atividades físicas e o outro com pacientes sedentárias. Dessa forma, analisar-se-á a influência do exercício físico associado à estimulação elétrica. 0 tratamento será de 20 sessões realizadas duas vezes por semana, utilizando-se a Tens com eletrodo tipo agulha $0.25 \times 30$, disposto abaixo da cicatriz umbilical. Pode-se ter como desconforto a corrente utilizada, a utilização das agulhas e estar semidespida na realização do tratamento, não existindo nenhum risco para as pacientes.

As informações obtidas por meio deste estudo serão tratadas rigorosamente com confidencialidade. Os resultados desta pesquisa serão divulgados publicamente; entretanto, sua identidade jamais será revelada. A sua participação neste estudo é voluntária. Você pode recusar-se a participar ou parar de participar a qualquer momento da pesquisa.

Li e entendi as informações procedentes descrevendo este assunto, e todas as minhas dúvidas em relação ao estudo e à minha participação nele foram respondidas satisfatoriamente. Dou livremente meu consentimento em participar do estudo até que decida pelo contrário.

Assinando este termo de consentimento concordo em participar deste estudo e não abro mão, na condição de participante de um estudo de pesquisa, de nenhum dos direitos legais que eu teria de outra forma. 


\section{Anexo 2}

\section{Questionário de prática habitual de atividade física proposto por Baecke}

\section{Seção 1 - Atividade na escola e no trabalho}

Questão 1 - Sua principal ocupação profissional

Questão 2 - Para realizar as atividades em seu trabalho você permanece sentado:

(1) nunca (2) raramente (3) algumas vezes (4) frequentemente (5) sempre

Questão 3 - Para realizar as atividades em seu trabalho você permanece em pé:

(1) nunca (2) raramente (3) algumas vezes (4) frequentemente (5) sempre

Questão 4 - Para realizar as atividades em seu trabalho você necessita caminhar:

(1) nunca (2) raramente (3) algumas vezes (4) frequentemente (5) sempre

Questão 5 - Para realizar as atividades em seu trabalho você necessita carregar cargas:

(1) nunca (2) raramente (3) algumas vezes (4) frequentemente (5) sempre

Questão 6 - Após um dia de trabalho você se sente cansado ou fatigado:

(1) nunca (2) raramente (3) algumas vezes (4) frequentemente (5) sempre

Questão 7 - Para realizar as atividades em seu trabalho você transpira:

(1) nunca (2) raramente (3) algumas vezes (4) frequentemente (5) sempre

Questão 8 - Em comparação com o trabalho de outras pessoas da mesma idade, você acredita que seu trabalho tenha esforço físico :

(1) nunca (2) raramente (3) algumas vezes (4) frequentemente (5) sempre

\section{Seção 2 - Atividades esportivas, programas de exercícios físicos e lazer ativo}

Questão 9 - Você pratica algum esporte ou está envolvido em programas de exercícios físico?

( ) $\operatorname{sim}$ ( ) não

Caso não pratique algum tipo de esporte/ programa de exercício físico, ir para questão 10.

Questão 9.1 - Como primeira opção, o esporte/programa de exercícios físicos que você mais frequentemente pratica apresenta intensidade:

( ) baixa ( ) moderada ( ) elevada

Questão 9.2 - Durante quantas horas/semanas você pratica esse esporte/programa de exercícios físicos?
()$<1 h$
( ) $1-2 \mathrm{~h}$
( ) $2-3 \mathrm{~h}$
( ) $3-4 h$
( ) $>4 \mathrm{~h}$

Questão 9.3 - Durante quantos meses/ano você pratica esse esporte/programa de exercícios físicos? ( ) $<1$ mês ( ) 1-3 meses ( ) 4-6 meses ( ) 7-9 meses ( ) $>9$ meses. 
Questão 9.4 - Caso você apresente uma segunda opção à prática de esporte/programa de exercícios físicos, ela é de intensidade:

( ) baixa ( ) moderada ( ) elevada

Caso não exista uma segunda opção à prática de esporte/programa de exercícios físicos, ir para questão 10.

Questão 9.5 - Durante quantas horas/semanas você pratica esse esporte/programa de exercícios físicos?
()$<1 \mathrm{~h}$
( ) $1-2 \mathrm{~h}$
( ) $2-3 h$
( ) $3-4 \mathrm{~h}$
()$>4 \mathrm{~h}$

Questão 9.6 - Durante quantos meses você pratica esse esporte/programa de exercícios físicos?

( ) $<1$ mês ( ) 1-3 meses ( ) 4-6 meses ( ) 7-9 meses ( ) $>9$ meses

Questão 10 - Em comparação com outras pessoas de mesma idade, você acredita que as atividades que realiza durante seu tempo livre são fisicamente:
(5) muito elevadas
(4) elevadas
(3) iguais
(2) baixas
(1) muito baixas

Questão 11 - Nas atividades de lazer e ocupação do tempo livre você transpira:

(5) muito frequentemente (4) frequentemente (3) algumas vezes ( 2 ) raramente (1) nunca

Questão 12 - Nas atividades de lazer e de ocupação do tempo livre você pratica esportes:

(1) nunca (2) raramente (3) algumas vezes (4) frequentemente (5) sempre

\section{Seção 3 - Atividades de ocupação do tempo livre}

Questão 13 - Nas atividades de lazer e de ocupação do tempo livre você assiste à TV:

(1) nunca (2) raramente (3) algumas vezes (4) frequentemente (5) sempre

Questão 14 - Nas atividades de lazer e de ocupação do tempo livre você caminha:

(1) nunca (2) raramente (3) algumas vezes (4) frequentemente (5) sempre

Questão 15 - Nas atividades de lazer e de ocupação do tempo livre você anda de bicicleta:

(1) nunca (2) raramente (3) algumas vezes (4) frequentemente (5) sempre

Questão 16 - Durante quanto tempo por dia você caminha e/ou anda de bicicleta para ir ao trabalho, à escola e às compras?

(1) < 5 min (2) 5-15 min (3) 15-30 min (4) 30-45 min (5) > 45 min 


\section{Anexo 3}

\subsection{Ficha de Avaliação}

\section{IDENTIFICAÇÃO:}

Nome:

Sexo: Tel. Res:

Nascimento:

Endereço:

Bairro: Cidade:

Est: Cep:

Profissão:

Motivo Da Consulta/Queixa Principal:

\section{HISTÓRIA DA DOENÇA ATUAL :}

Desde quando persiste o problema?

Pode-se relacionar o problema com alguma circunstância?

Realizou algum tratamento anteriormente?

Como foram os resultados do tratamento realizado?

\section{HÁBITOS DE VIDA}

Atividade cotidiana (sedentário ou ativo)

Sono (horas diárias)

Tabagismo (cigarros/dia)

Etilismo (quantidade)

Estado emocional (ansiedade, nervosismo, estressado, depressão, etc.)

Cuidados corporais habituais (que tipo? Frequência?)

Cuidados faciais habituais (que tipo? Frequência?)

Indumentária (utilização de faixa, ligas ou meios oclusivos)

Exposição ao tempo/clima (qual? Com que frequência?)

Outras anotações relevantes:

\section{HISTÓRIA PATOLÓGICA PREGRESSA}

Enfermidades anteriores

Enfermidades atuais

Medicação habitual (corticoides /anti-histamínicos/esteroides/AINH/etc.)

Alterações hormonais:

Alergias

Diabetes

Propensão a queloides: $\operatorname{SIM}($ ) NÃO ( )

Hemofilia

Marca-passos cardíacos 
Intervenções cirúrgicas: cirurgia plástica lifting blefaroplastia riboplastia lipoaspiração dermolipectomia (abdominoplastia) mamoplastia de redução rejuvenescimento facial mamoplastia de aumento lipoescultura mastectomia microcirurgia mentoplastia otoplastia/rinosseptoplastia implante de silicone aplicação de botox

\section{ANTECEDENTES FAMILIARES}

\section{FATORES ALIMENTÍCIOS}

Tipo de alimentação (normal, vegetariano, etc.)

Quantidade normal pouca excessiva

Quantidade de refeições ao dia: $01 \quad 02 \quad 03 \quad 04$ outras

Quantidade de líquidos ao dia

Alterações digestivas

Dieta fez faz

Obesidade Sim Não Endógena Exógena

\section{HISTORIA GINECOLÓGICA}

Idade da $1^{\underline{a}}$ menstruação

Alterações no fluxo menstrual (regularidade, quantidade, etc.)

Tensão pré-menstrual

Quantidade de gestações

Método anticonceptivo

\section{EXPLORAÇÃO FÍSICA}

Data

Idade Peso Altura

Pressão Arterial

Perimetria:

Cintura:

$10 \mathrm{~cm}$ acima da crista ilíaca:

$5 \mathrm{~cm}$ acima da crista ilíaca:

Crista ilíaca:

IMC

C/Q:

\section{REAVALIAÇÃO}

Data:

Peso

Perimetria:

Cintura:

$10 \mathrm{~cm}$ acima da crista ilíaca:

$5 \mathrm{~cm}$ acima da crista ilíaca:

Crista ilíaca:

IMC

C/Q: 
Melo NR, Monteiro FMAC, Pontes GAR, Mello SMB.

\section{REAVALIAÇÃO}

Data:

Peso

Perimetria:

Cintura:

$10 \mathrm{~cm}$ acima da crista ilíaca:

$5 \mathrm{~cm}$ acima da crista ilíaca:

Crista ilíaca:

IMC 